Tohoku J. Exp. Med., 2011, 223, 125-131

\title{
Repeat Stereotactic Radiosurgery in the Management of Brain Metastases from Non-Small Cell Lung Cancer
}

\author{
Yasushi Mariya, ${ }^{1}$ Genichirou Sekizawa, ${ }^{1}$ Yoshisuke Matsuoka, ${ }^{1}$ Hirobumi Seki, ${ }^{2}$ \\ Takayuki Sugawara ${ }^{2}$ and Yuka Sasaki ${ }^{3}$ \\ ${ }^{1}$ Department of Radiation Oncology, Iwate Prefectural Central Hospital, Morioka, Japan
2 Department of Neurosurgery, Iwate Prefectural Central Hospital, Morioka, Japan
${ }^{3}$ Department of Psychiatry, Iwate Prefectural Central Hospital, Morioka, Japan
}

Non-small cell lung cancer (NSCLC) is characterized by brain metastases that occur in about 30 to $50 \%$ of patients. To control tumor growth potential with maintaining neurocognitive function is important in the recent radiotherapy against brain metastases. From this viewpoint, we investigated the utility of repeat stereotactic radiosurgery (SRS) with a linear accelerator in the management of brain metastases from NSCLC. Between October 1998 and May 2010, 28 patients harboring brain metastases received repeat SRS (20 men and 8 women, with the age ranged from 51 to 79 ). The total number of SRS sessions ranged from 2 to 5 , and the total number of lesions in one patient ranged from 1 to 8 . Neurological decline due to uncontrolled brain lesions was identified in 9 of 28 patients after the repeat SRS, while the remaining 19 patients showed no neurological decline. Out of the 28 patients, 18 patients died by July 1, 2010; 12 patients died of active extracranial disease and 6 patients died from progressive brain lesions, considered neurological death. The 2-year and 4-year overall survival rates were $51 \%$ and $23 \%$, respectively, and the median survival time was 26 months. In conclusion, repeat SRS is a preferred option to manage brain metastases from NSCLC, leading to a long survival with a decreased neurological decline. Repeat SRS is promising to preserve neurocognition, because the convergent dose distribution decreases the unfavorable influences from radiation on germinal niches, thereby preserving neural stem cells that are responsible for the nervous system repair.

Keywords: repeat stereotactic radiosurgery; brain metastasis; non-small cell lung cancer; neurocognitive function; neural stem cell

Tohoku J. Exp. Med., 2011, 223 (2), 125-131. C 2011 Tohoku University Medical Press

It is well known that, in about 30 to $50 \%$ of patients associated with non-small cell lung cancer (NSCLC), brain metastases occur at some time during the clinical course of their disease and frequently cause their death (Sorensen et al. 1988; Lagerwaad et al. 1999; Wen and Loeffler 1999; Schoutten et al. 2002). Management of brain lesions is crucial for prolonging their survival, and avoiding neurological decline due to uncontrolled brain lesions is important to maintain their quality of life (QOL). In this regard, many reports have revealed that stereotactic radiosurgery (SRS) provides great benefits in managing brain metastases (Loeffler and Alexander 1993; Sneed et al. 2002; Mehta et al. 2005; Serizawa 2009; Linsky et al. 2010). Recently, we also demonstrated the clinical usefulness of SRS using a linear accelerator to control brain metastases from NSCLC and to avoid neurological death (Mariya et al. 2010).

On the other hand, recent reports (Aoyama et al. 2007; Chang et al. 2009) revealed that whole brain radiation therapy (WBRT), which had been the mainstay of treatment to brain metastases for many years, had unfavorable influences on neurocognitive function (NCF) which correlated well with the decreased QOL ( $\mathrm{Li}$ et al. 2008). Therefore, radiotherapeutic techniques, which have a potential to control recurrent and/or newly developed brain metastases with avoiding neurocognitive impairment, are being required.

In the current study, we focused on the patients with brain metastases from NSCLC who underwent repeat SRS using a linear accelerator and analyzed the outcome to assess the clinical utility and the radiobiological implication of the treatment option, repeat SRS.

\section{Patients and Methods}

Patients

Between October 1998 and May 2010, 97 patients with histoor cytopathologically proven NSCLC metastatic to the brain under-

Received November 30, 2010; revision accepted for publication January 15, 2011. doi: 10.1620/tjem.223.125

Correspondence: Yasushi Mariya, Department of Radiation Oncology, Iwate Prefectural Central Hospital, 1-4-1 Ueda, Morioka, Iwate

020-0066, Japan.

e-mail: ymariya@pref.iwate.jp 
Table 1. Baseline characteristics of patients.

\begin{tabular}{ll}
\hline Characteristics & \\
\hline Age (year) & Range 51-79, Median 67 \\
Gender & Male 20, Female 8 \\
Performance status & $0: 12,1: 10,2: 6$ \\
Histopahological status & ADCA 13, SCC 5, Large 5, NSCLC 5 \\
Presence of active extracranial lesion & Yes 17, No 11 \\
Previous treatment to lung primary & Yes 20, No 8 \\
Interval between Tx-primary and Ini-SRS (months) & Range 0-99, Median 10 \\
RTOG RPA Classification* & Class 1: 4, Class 2: 17, Class 3: 7 \\
\hline
\end{tabular}

ADCA, adenocarcinoma; SCC, squamous cell carcinoma; Large, large cell carcinoma; NSCLC, nonsmall cell lung carcinoma; Tx-primary, treatment to lung primary; Ini-SRS, initial session of stereotactic radiosurgery; RTOG, Radiation Therapy Oncology Group; RPA, recursive partitioning analysis (Gasper et al. 1997). ${ }^{*}$ Class 1: KPS $=>70$, aged $<65$ years, and no active extracranial disease; Class 3 : KPS $<70$, Class 2: all others.

went SRS at Iwate Prefectural Central Hospital. They included 28 patients that received repeat SRS as salvage therapy, performed twice or more to brain metastases. There were 20 men and 8 women, and the median age was 67 years (range, 51-79). The number of patients with baseline performance status (PS) 0,1 and 2 was 12,10 and 6, respectively. Histopathological subtype distribution for the lung primary included the following: 13 adenocarcinomas, 5 squamous cell carcinomas, 5 large cell carcinomas and 5 unclassified NSCLC. Out of the 28 patients, $20(71 \%)$ presented with newly developed distant brain metastasis outside the initial SRS volume, 5 (18\%) with progression of the original tumor uncontrolled by initial SRS, and 3 $(11 \%)$ with both. The maximum tumor diameter ranged from 4 to 34 $\mathrm{mm}$. For the lung primary, the 28 were comprised of 8 with previously untreated stage IV disease and 20 recurrent or relapsed ones that were definitely treated with surgery or chemoradiotherapy (CRT). Treatment modalities for the lung primary of the former 8 patients were the following: 2, CRT and 6, chemotherapy. Chemotherapy, alone or in combination with irradiation, was administered chiefly with platinum-based regimens. The interval between the date of start to treat the lung primary and the date of initial SRS ranged from 0 to 99 months (median, 10 months). The number of patients with active extracranial disease, including previously untreated stage IV disease, and that without active extracranial disease was 17 and 11, respectively. According to the Radiation Therapy Oncology Group (RTOG) recursive partitioning analysis classification (Gasper et al. 1997), the patients were classified as follows: 4 in Class 1, 17 in Class 2, and 7 in Class 3. The baseline characteristics of the patients are listed in Table 1. For repeat SRS, patients were considered to be eligible when the number of brain metastases was limited within 4, the PS 0-2, and the expected prognosis 3-4 months or longer.

\section{Treatment}

SRS was administered with a linear accelerator (CLINAC 2100C, Varian Medical Systems, Palo Alto, CA, USA), equipped with an F.L. Fischer Stereotactic Radiosurgery System (Leibinger, Heidelberg, Germany), and 10 MV X-ray was used. The SRS system was comprised of rigid head immobilization devices, a set of collimators of various aperture sizes, a CT localizer, target positioning devices for the linear accelerator, target point verification devices using portal films, and a computerized radiotherapy planning system.
When planning SRS, patients had a contrast enhancing CT scan; gross target volume (GTV), equaled the clinical target volume, was defined as the contrast-enhanced tumor volume. Planning target volume (PTV) was defined as the volume, GTV with a 1 to $2 \mathrm{~mm}$ margin of surrounding brain tissue. The six-arc treatment plan with a single isocenter was employed, except for an ellipsoidal and large target lesion for which a two-isocenter plan was needed. The dose for initial SRS, delivered in a single fraction and prescribed to the isocenter, ranged from 15 to $33 \mathrm{~Gy}$ (median, $25 \mathrm{~Gy}$ ). The marginal dose to PTV margin, corresponding to about $80 \%$ of the isocenter dose, ranged from 12 to $20 \mathrm{~Gy}$ (median, $20 \mathrm{~Gy}$ ). When there were multiple target lesions in a single session, independent SRS was planned and delivered to each of them within the same treatment day.

For the 23 patients with newly developed metastases, repeat SRS was delivered in the same manner as the initial one with an isocenter dose of $25 \mathrm{~Gy}$ and the respective marginal dose of $20 \mathrm{~Gy}$. For the 8 patients with locally uncontrolled tumors, it was delivered with an isocenter dose which ranged from 18 to 25 Gy (median, 20 Gy), mostly reduced in comparison to that for the initial one, and the respective marginal dose ranged from 12 to $20 \mathrm{~Gy}$ (median, $16 \mathrm{~Gy}$ ). Further, 2 of them received a third session of SRS at the same site. The total number of SRS sessions was as follows: 2 in 21 patients, 3 in 4 patients, 4 in 2 patients, and 5 in 1 patient (median, 2 and mean, 2.4). The number of target lesions at each session was as follows: 1-3 at initial SRS, 1-4 at the second one, 1-2 at the third one, 1 at the fourth one, and 1 at the fifth one as shown in Fig. 1. The total number of target lesions in one patient ranged from 1 to 8 (median, 3 and mean, 3.1).

Twelve patients underwent other modalities as an additional salvage therapy following repeat SRS: 6 , WBRT and 6, surgery. WBRT was delivered with a total target dose of 30 Gy in 10 fractions or $40 \mathrm{~Gy}$ in 16 fractions to intracranially distributed metastases and/ or carcinomatous meningitis in the advanced stage of their clinical course. Of the 6 who underwent surgery as an additional salvage, 4 received resection of tumor or tumor-suspected lesion after repeat SRS at the original site. The remaining 2 received ventricle-peritoneum shunting after repeat SRS because of progressive hydrocephalus due to the mass effect from extended tumor. 


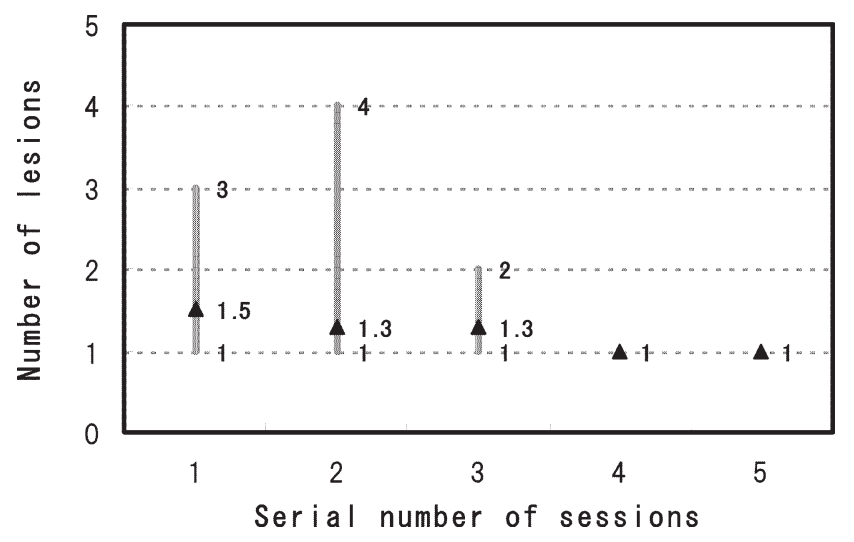

Fig. 1. Number of target lesions at each session of stereotactic radiosurgery.

Closed triangles and bars represent mean and range of number of target lesions at each session of stereotactic radiosurgery (SRS), respectively. Number 1 in "Serial number of sessions" represents initial session of SRS. Numbers 2 to 5 represent repeat sessions of SRS.

\section{Follow-up and Statistical analysis}

The patients were followed clinically and by serial MR imaging and CT scanning performed at least every 3 months. Neurological status was retrospectively estimated from the medical records, according to the criteria by Bhatnagar et al. (2002), based on the serial change of 3 neurological symptoms: seizures, focal deficits, and headaches. An increase in any of the 3 neurological symptoms or a new symptom after repeat SRS was considered to indicate neurological decline. Although in a limited number of patients, NCF was examined by using the revised version of Hasegawa's Dementia Scale (HDS-R) (Katou et al. 1991). The maximum score that can be obtained for the entire HDS-R is 30 points. If the score of HDS-R is below 20 points, then the patient can be classified as having dementia. The median follow-up period after initial SRS was 15 months (range, 4-54), and that of post-repeat SRS after second SRS was 6.5 months (range, 1-49). Time in months of overall survival (OS) was computed from the date of the patient's initial SRS, and, for the post-repeat SRS, from the date of second SRS. Curves of survival and median survival time (MST) were calculated using the Kaplan-Meier method. All analyses were carried out using the statistical software package SPSS 11.0J for Windows (SPSS Japan Inc., Tokyo).

\section{Results}

\section{Patient survival}

The 2- and 4-year OS rates were $51 \%$ and $23 \%$, respectively (Fig. 2), and the MST was 26 months. The 2and 4 -year OS rates after the repeat SRS were $31 \%$ and $13 \%$, respectively (Fig. 3), and the MST of post-repeat SRS was 11 months.

\section{Cause of Death and neurological death}

Until July 1, 2010, 18 out of 28 patients (64\%) died. Twelve patients died of active extracranial disease (12/18, $67 \% ; 12 / 28,43 \%$ ): uncontrolled thoracic lesions and/or systemic spreads other than brain lesions. When the patient died from progressive brain lesions, either within or out of the SRS volume, cause of death was considered neurologi-

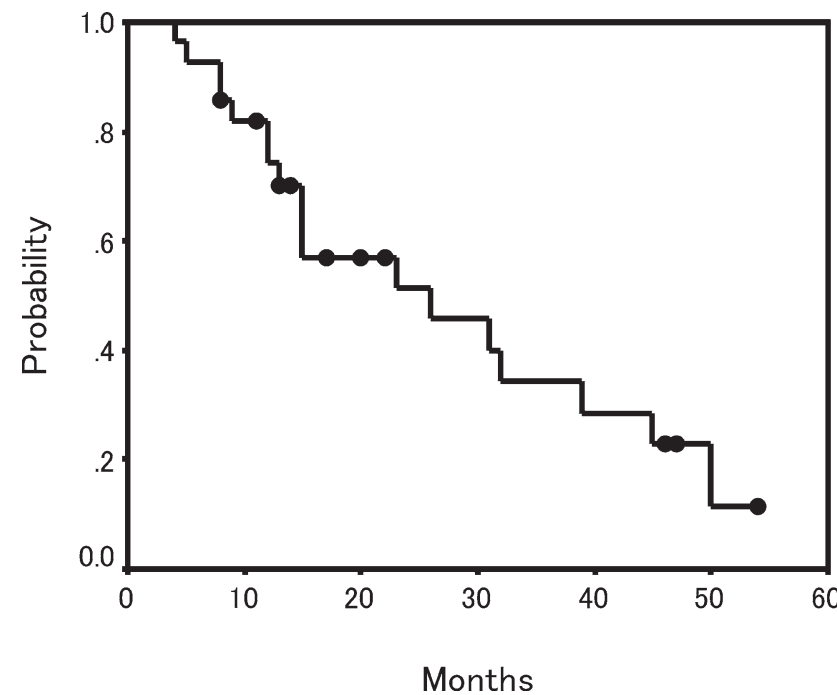

Fig. 2. Kaplan-Meier curve of overall survival for all 28 patients.

The 2- and 4-year overall survival rates were $51 \%$ and $23 \%$, respectively. The median survival time was 26 months.

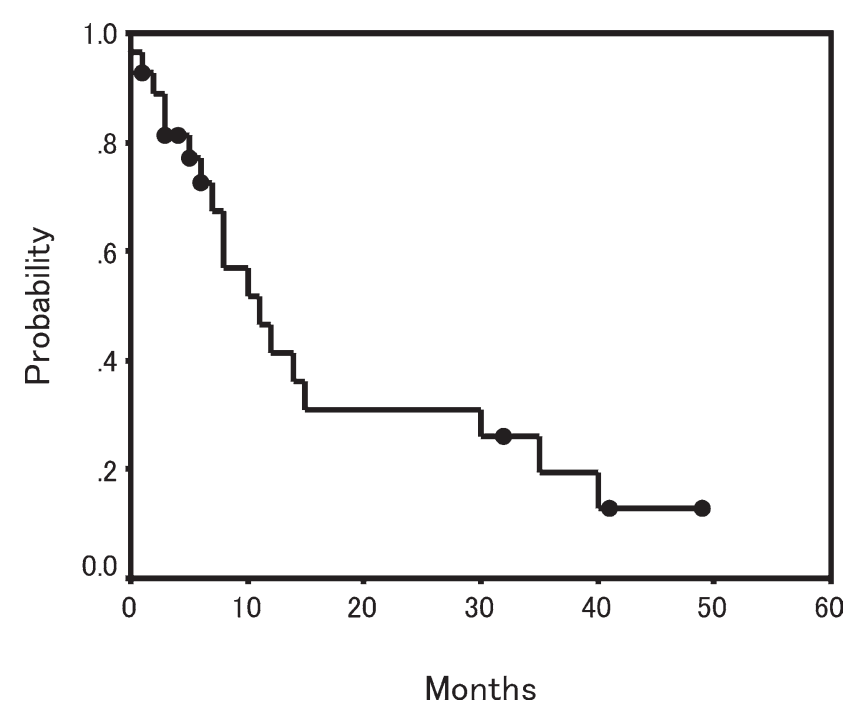

Fig. 3. Kaplan-Meier curve of overall survival for post-repeat stereotactic radiosurgery $(n=28)$.

Time in months of overall survival (OS) for post-repeat stereotactic radiosurgery (SRS) was computed from the date of second session of SRS or the start date of repeat SRS. The 2- and 4-year OS rates for post-repeat SRS were $31 \%$ and $13 \%$, respectively. The median survival time for that was 11 months.

cal. The neurological death was determined in 6 patients $(6 / 18,33 \% ; 6 / 28,21 \%)$. The 2 - and 4 -year rates of patients free from neurological death were $81 \%$ and $56 \%$, respectively (Fig. 4).

\section{Neurological status and neurocognitive function}

Neurological decline according to the criteria by Bhatnagar et al. (2002) was identified in 9 out of 28 (32\%). Six of the 9 patients with uncontrolled brain lesions resulted 
in neurological deaths, as aforementioned. The remaining 3 patients presented with neurological decline due to uncontrolled brain lesions, although they died of active extracranial disease: extensive progression of thoracic lesions. No neurological decline was identified in the other 6 patients who died of active extracranial disease.

In 10 patients alive with controlled brain lesions, no apparent neurological decline was observed. For 5 patients, the examination of HDS-R was carried out in the most recent follow-up (Table 2). The interval between initial SRS and the examination of HDS-R ranged from 8 to 48 months (median, 20 months). The number of repeat SRS sessions ranged from 2 to 3 (median, 2), and the total number of target lesions ranged from 2 to 6 (median, 3). All of their HDS-R scores exceeded 20 points, ranging from 25 to 30 points (median, 26 points), which meant none of the 5 patients were in the status of dementia.

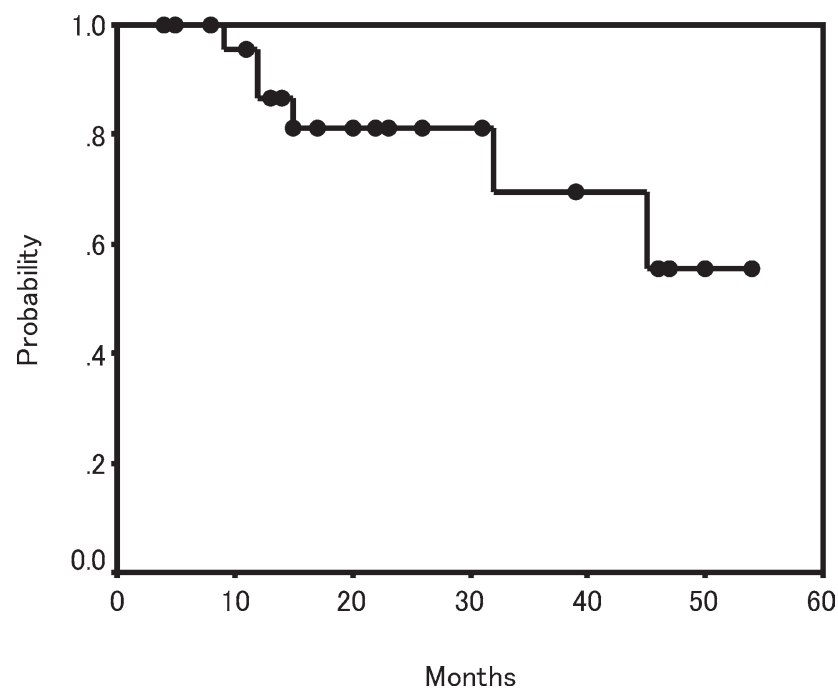

Fig. 4. Kaplan-Meier curve of patients free from neurological death $(n=28)$.

The 2- and 4-year rates of patients free from neurological death due to progressive brain lesions were $81 \%$ and $56 \%$, respectively.

\section{Major complications}

No major acute toxicity of central nervous system (CNS) was detected from the medical records (Radiation Therapy Oncology Group). As to chronic CNS toxicity, one patient underwent surgical resection for localized but symptomatic radiation necrosis after 3 repeat sessions of SRS at an original site, initial SRS followed by 2 sessions as salvage. Another patient with a similar course of treatment also underwent surgical resection, whereas the radiation necrosis-suspected lesion was revealed histopathologically to be necrosis mixed with a small part of tumor growth. Only these 2 patients who received SRS repeated three times at the same site were recognized to have a major chronic CNS toxicity of grade 4 according to the RTOG/ EORTC scoring schema (Radiation Therapy Oncology Group). No other major chronic CNS toxicity was identified from the records of the remaining 26 patients.

\section{Discussion}

At present, no consensus on treatment exists for the patients who survive long enough to experience recurrence and/or progression of previously treated brain metastases (Ammirati et al. 2010). Although there still have been few studies that demonstrated the clinical utility of repeat SRS for brain metastases, Yamanaka et al. (1999) and Shuto et al. (2004) reported relatively long survivals in the patients for whom repeat SRS was employed for recurrent and/or newly developed brain metastases originating from various types of malignancies: the MST from initial SRS was 15 and 22.4 months, respectively. Further, Chen et al. (2000) and Kwon et al. (2007) reported that the MST calculated from the date of SRS as retreatment was 7 and 8 months, respectively, in the patients whose initial treatment included SRS for brain metastases originating from various types of malignancies.

The results shown in the current study, the MST, 26 months and that for post-repeat SRS, 11 months, were comparable or better than those in the abovementioned studies. Thus, our results appear to be quite good, considering that

Table 2. Repeat stereotactic radiosurgery and neurological status in 5 patients.

\begin{tabular}{|c|c|c|c|c|c|c|c|c|c|c|c|c|c|c|}
\hline \multirow{2}{*}{ No. } & \multirow{2}{*}{ Gender } & \multirow{2}{*}{ Age } & \multirow{2}{*}{ PS } & \multirow{2}{*}{ ECL } & \multirow{2}{*}{ Class } & \multirow{2}{*}{$\begin{array}{l}\text { Total } \\
\text { SRS }\end{array}$} & \multicolumn{3}{|c|}{ Session \& Target-No. } & \multirow{2}{*}{$\mathrm{PS}_{2 \mathrm{nd}}$} & \multirow{2}{*}{ ND } & \multirow{2}{*}{ HDS- $\mathrm{R}^{* *}$} & \multirow{2}{*}{$\begin{array}{c}\text { Period } \\
\text { (M) }\end{array}$} & \multirow{2}{*}{ Remarks } \\
\hline & & & & & & & 1 & 2 & 3 & & & & & \\
\hline 1 & M & 72 & 1 & No & 2 & 2 & 3 & $1(1)^{*}$ & & 2 & No & 26 & 48 & Died of ECL $(50 \mathrm{M})$ \\
\hline 2 & M & 80 & 1 & No & 2 & 3 & 2 & 2 & 2 & 1 & No & 30 & 22 & \\
\hline 3 & F & 59 & 0 & No & 2 & 2 & 1 & 2 & & 0 & No & 28 & 20 & \\
\hline 4 & M & 68 & 1 & No & 2 & 3 & 1 & 1 & $1(1)^{*}$ & 1 & No & 25 & 17 & \\
\hline 5 & M & 73 & 0 & No & 2 & 2 & 2 & 3 & 1 & 0 & No & 25 & 8 & \\
\hline
\end{tabular}

M, male; F, female; PS, performance status; ECL, active extracranial lesion; Class, Class according to Radiation Therapy Oncology Group recursive partitioning analysis classification (Gasper et al. 1997); Total SRS, total number of stereotactic radiosurgery (SRS) sessions; Session \& Target-No., Order of SRS sessions and number of target lesions in each session; PS2nd, PS at the time of examination using revised version of Hasegawa's Dementia Scale (HDS-R) (Katou et al. 1991); ND, neurological decline defined according to the criteria by Bhatnagar et al. (2002); Period, period between initial SRS and the time of examination using HDS-R; M, months. *In parenthesis is number of SRS sessions performed at the same site. **Number represents the score of HDS-R $(\max .=30)$. 
our patients were limited to those with brain metastases from NSCLC usually having a very poor prognosis. In addition, the number of patients whose outcome resulted in neurological death or who had neurological decline due to tumor progression was relatively fewer than expected, meaning that the QOL in most of the patients was wellpreserved. One of the reasons why our results were relatively good may be careful clinical monitoring, consisting of neurological examination and diagnostic images performed at least every 3 months, which is essential when the strategy of SRS allowing for repeat SRS as salvage is taken to treat brain metastases (Aoyama et al. 2006; Chang et al. 2009). As a result, very small tumors can be detected on images even without neurological symptoms and signs, when the local recurrence and/or newly developed metastases occur in the brain. Consequently, the small target volume leads to not only good tumor control but also increased tolerability of normal brain tissue to radiation, the most important limiting factor in SRS (Withers et al. 1988; Flickinger et al. 1990; Lawrence et al. 2010).

As to tolerance of previously irradiated brain tissue when using SRS as a retreatment after failure, there has been limited data (Ammirati et al. 2010). However, it is of note that Shaw et al. (2000) did report the maximum tolerated dose of single fraction SRS in patients with recurrent primary brain tumors and brain metastases previously treated by partial or whole brain fractionated external beam radiotherapy (EBRT). They concluded that the maximum tolerated doses of single fraction SRS for the above population of patients were defined as $24 \mathrm{~Gy}, 18 \mathrm{~Gy}$, and $15 \mathrm{~Gy}$ for tumors with a maximum diameter of $\leq 20 \mathrm{~mm}, 21-30$ $\mathrm{mm}$, and 31-40 mm, respectively. Moreover, Bhatnagar et al. (2002) employed repeat SRS for progressive primary and metastatic CNS tumors and showed that the treatment volume for repeat SRS was the only variable significantly correlated with neurological decline. Those reports demonstrated that, when using SRS as a retreatment, tumor size or treatment volume, as well as radiation dose, was critical for the tolerance or neurological decline of previously irradiated brain tissue either with EBRT or SRS.

From our results, normal brain tissue appeared clinically tolerable to SRS repeated two times for locallyrecurrent tumor at an original site. However, symptomatic radiation necrosis or chronic CNS toxicity of grade 4 was observed in the 2 patients who received SRS repeated three times at the original site, which meant that the accumulated radiation effects were excessive and caused severe brain tissue damage. Although the number of patients was very few, at least it was suggested that repetition of SRS at the same site should be limited to two times. It is vital that we pay more attention to the indication when repeating SRS to the same lesion, as Chin et al. (2001) noted.

Concerning the function of damaged brain tissue at the site of SRS, it is difficult to expect the preservation of local tissue viability after high dose irradiation by SRS. Both metastatic tumor and SRS itself destroy the brain tissue, resulting in a local loss of function. However, except for the eloquent regions, a large proportion of brain tissue is regarded as a "parallel organ" (Abe and Aoki 2002), which can be expected to maintain the function as a whole brain, irrespective of a local functional loss at the site of SRS. In addition, we previously experienced the serial improvement of electroencephalogram findings in the patients associated with primary brain tumor treated with surgery and postoperative fractionated EBRT, which suggested the brain plasticity or the capability of functional compensation by surviving and surrounding brain tissues (Mariya et al. 1999). Thus, it can be speculated that, regarding repeat SRS at the same site, if the treatment volume is sufficiently localized and almost unchanged in size compared with that of the initial SRS while avoiding eloquent regions, then an additional loss of function leading to neurological decline may be minimal or not manifest. In this regard, we have not observed clinically apparent neurological decline in the 16 patients other than that associated with intracranial tumor progression. Also the scores of HDS-R in the 5 patients examined revealed that none of them was classified as having impaired neurocognition. These results suggested the functional safety of repeat SRS.

Moreover, we would like to refer to the radiobiological implication of SRS in contrast to WBRT, from the viewpoint of treatment volume and preservation of neural stem cells (NSCs) (Barani et al. 2007a, 2007b). Recently, Aoyama et al. (2006) reported the results of a prospective, randomized controlled trial comparing upfront WBRT of 30 Gy in 10 fractions plus SRS versus SRS alone for patients with a limited number $(\leq 4)$ of brain metastases. They showed a significantly better local tumor control and prophylactic effects outside the SRS volume in the upfront WBRT plus SRS group compared with that in the SRS alone group, although there was no significant survival difference between the 2 groups. At the same time, they compared the NCF of patients harboring brain metastasis who received upfront WBRT plus SRS with SRS alone (Aoyama et al. 2007). To monitor NCF, they serially used the MiniMental State Examination, the same kind of examination as HDS-R, and noted that WBRT could be a cause of continuous neurocognitive deterioration in long-term survivors. Furthermore, Chang et al. (2009) demonstrated that patients treated with SRS plus WBRT were at a greater risk of a significant decline in learning and memory function by 4 months in comparison to the patients who received SRS alone.

These studies offered evidence that upfront WBRT in combination with SRS was associated with impaired neurocognition. In order to understand the mechanism of neurocognitive impairment related to WBRT, applying the concept of treatment-induced NSC dysfunction is suitable (Barani et al. 2007a, 2007b). WBRT includes the supratentorial brain as a large proportion of target volume, in which the subventricular zone (SVZ) of the lateral ventricles and the subgranular zone (SGZ) of the hippocampal dentate 
gyrus are located. Both of the SVZ and the SGZ are recognized as major germinal niches in adults, which contain NSCs capable of driving neurogenesis and gliogenesis, and are central to nervous-system repair and preservation and to reconstitution of brain function by migration and integration of neuronal and glial cells derived from the NSCs. Radiation can directly depopulate the NSC niches, causing immediate loss of NSC-mediated repair and plasticity in brain tissue, and can indirectly have an adverse effect on normal proliferation and differentiation of surviving NSCs from inflammation-mediated changes in the microenvironment (Barani et al. 2007a). The fundamental findings suggest that brain radiotherapy should be delivered in a NSCpreserving manner as much as possible. In this context, SRS alone can be offered as an adequate radiation technique to manage brain metastases and preserve brain function, because the unfavorable influences from radiation on the NSC niches can be excluded or minimized by the convergent dose distribution to target lesions. In the current study, the influence from WBRT, added as a final salvage therapy for the 6 patients in the advanced stage, could not be determined because these patients died soon thereafter.

Finally, we would like to emphasize that SRS allowing for repeat SRS as salvage, which has an excellent clinical potential to control tumor and preserve NSCs and brain function, should be considered as a preferred treatment option to brain metastases from NSCLC if indicated, particularly to those in the patients who are expected to have a long survival. Repeat SRS appears tolerable when adequately employed, for either a newly developed metastasis or a locally uncontrolled one, whereas it is essential to avoid excessive radiation to normal brain tissue around the metastatic tumor as much as possible.

\section{Conclusions}

Although careful clinical monitoring was needed, repeat SRS using linear accelerator in the management of locally-recurrent and/or newly developed brain metastases from NSCLC was an effective treatment option, leading to a long survival with a decreased neurological decline. Further, it was suggested that repeat SRS could play a crucial role as a relevant approach of NSC-preserving radiotherapy.

\section{Acknowledgments}

The authors would like to acknowledge the contributions of Ms. Shino Hirai in preparing the figures. The authors would also like to thank Paul Langman, Ph.D. for his careful reading of the manuscript and kind assistance with English usage.

\section{References}

Abe, Y. \& Aoki, M. (2002) Normal Tissue Reaction. In GanHoushasenryouhou 2002, edited by Ohkawa, T., Tanaka, Y. \& Sasaki, T. Shinohara Shuppan, Tokyo, pp. 142-146. (In Japanese)

Ammirati, M., Cobbs, C.S., Linskey, M.E., Paleologos, N.A., Ryken, T.C., Burri, S.H., Asher, A.L., Loeffler, J.S., Robinson,
P.D., Andrews, D.W., Gasper, L.E., Kondziolka, D., McDermott, M., Mehta, M.P., Mikkelsen, T., Olsen, J.J., Patchell, R.A. \& Kalkanis, S.N. (2010) The role of retreatment in the management of recurrent/progressive brain metastases: a systemic review and evidence-based clinical practice guideline. J. Neurooncol., 96, 85-96.

Aoyama, H., Shirato, H., Tago, M., Nakagawa, K., Toyoda, T., Hamano, K., Kenjyo, M., Oya, N., Hirota, S., Shioura, H., Kunieda, E., Inomata, T., Hayakawa, K., Katoh, N., \& Kobashi, G. (2006) Stereotactic radiosurgery plus wholebrain radiation therapy vs. stereotactic radiosurgery alone for treatment of brain metastases. JAMA, 295, 2482-2491.

Aoyama, H., Tago, M., Kato, N., Toyoda, T., Kenjyo, M., Hirota, S., Shioura, H., Inomata, T., Kunieda, E., Hayakawa, K., Nakagawa, K., Kobashi, G. \& Shirato, H. (2007) Neurocognitive function of patients with brain metastasis who received either whole brain radiotherapy plus stereotactic radiosurgery or radiosurgery alone. Int. J. Radiat. Oncol. Biol. Phys., 68, 1388-1395.

Barani, I.J., Benedict, S.H. \& Lin P.S. (2007a) Neural stem cells: implications for the conventional radiotherapy of central nervous system malignancies. Int. J. Radiat. Oncol. Biol. Phys., 68, 324-333.

Barani, I.J., Cuttino, L.W., Benedict, S.H., Todor, D., Bump, E.A., Wu, Y., Chung, T.D., Broaddus, W.C. \& Lin P.-S. (2007b) Neural stem cell-preserving external-beam radiotherapy of central nervous system malignancies. Int. J. Radiat. Oncol. Biol. Phys., 68, 978-985.

Bhatnagar, A., Heron, D.E., Kondziolka, D., Lunsford, L.D. \& Flickinger, J.C. (2002) Analysis of repeat stereotactic radiosurgery for progressive primary and metastatic CNS tumors. Int. J. Radiat. Oncol. Biol. Phys., 37, 745-751.

Chang, E.I., Wefel, J.S., Hess, K.R., Allen, P.K., Lang, F.K., Kornguth, D.G., Arbuckle, R.B., Swint, J.M., Shiu, A.S., Maor, M.H. \& Meyers, C.A. (2009) Neurocognition in patients with brain metastases treated with radiosurgery or radiosurgery plus whole-brain irradiation: a randomized controlled trial. Lancet Oncol., 10, 1037-1044.

Chen, J.C., Petrovich, Z., Giannota, S.I., Yu, C. \& Appuzo, M.L. (2000) Radiosurgical salvage therapy for patients presenting with recurrence of metastatic disease to the brain. Neurosurgery, 46, 860-866.

Chin, L.S., Ma, L. \& DiBiase, S. (2001) Radiation necrosis following gamma knife surgery: a case-controlled comparison of treatment parameters and long-term clinical follow up. $J$. Neurosurg., 94, 899-904.

Flickinger, J.C., Schell, M.C. \& Larson, D.A. (1990) Estimation of complications for linear accelerator radiosurgery with the integrated logistic formula. Int. J. Radiat. Oncol. Biol. Phys., 19, $143-148$

Gasper, L., Scott, C., Rotman, M., Asbell, S., Phillips, T., Wasserman, T., McKenna, W.G. \& Byhardt, R. (1997) Recursive partitioning analysis (RPA) of prognostic factors in three radiation therapy oncology group (RTOG) brain metastasis trials. Int. J. Radiat. Oncol. Biol. Phys., 37, 745-751.

Katou, S., Shimogaki, H., Onodera, A., Ueda, H., Oikawa, K., Ikeda, K., Kosaka, A., Imai, Y. \& Hasegawa, K. (1991) Development of the revised version of Hasegawa's dementia scale. Jpn. J. Geriatr. Psych., 2, 1339-1347. (In Japanese)

Kwon, K.Y., Kong, D.S., Lee, J.I., Nam, D.H., Park, K. \& Kim, J.H. (2007) Outcome of repeated radiosurgery for recurrent metastatic brain tumors. Clin. Nerol. Neurosurg., 109, 132137.

Lagerwaad, F.J., Levendag, P.C., Nowak, P.J.C.M., Eijkenboom, W.M.H., Hanssens, P.E.J. \& Schmitz, P.I.M. (1999) Identification of prognostic factors inpatients with brain metastases: a review of 1292 patients. Int. J. Radiat. Oncol. Biol. Phys., 43, 795-803.

Lawrence, Y.R., Li, X.A., Naqa, I.E., Hahn, C.A., Marks, L.B., 
Merchant, T.E. \& Dicker, A.P. (2010) Radiation dose-volume effects in the brain. Int. J. Radiat. Oncol. Biol. Phys., 76, S20S27.

Li, J., Bentzen, S.M., Li, J., Renschler, M. \& Mehta, M.P. (2008) Relationship between neurocognitive function and quality of life after whole-brain radiotherapy in patients with brain metastasis. Int. J. Radiat. Oncol. Biol. Phys., 71, 64-70.

Linsky, M.E., Andrews, D.W., Asher, A.L., Burri, S.H., Kondziolka, D., Robinson, P.D., Ammirati, M., Cobbs, C.S., Gasper, L.E., Loeffler, J.S., McDermott, M., Mehta, M.P., Mikkelsen, T., Olsen, J.J., Paleologos, N.A., Patchell, R.A., Ryken, T.C. \& Kalkanis, S.N. (2010) The role of stereotactic radiosurgery in the management of patients with newly diagnosed brain metastases: a systematic review and evidencebased practice guideline. J. Neurooncol., 96, 45-68.

Loeffler, J.S. \& Alexander, E. (1993) Radiosurgery for the treatment of intracranial metastases. In Stereotactic Radiosurgery, edited by Alexander, E., Loeffler, J.S., Lunsford, D., pp. 197-206.

Mariya, Y., Saito, F., Kimura, T., Aoki, M., Anbai, A., Basaki, K., Matsukura, H., Abo, M., Kondou, H. \& Abe, Y. (1999) Cerebral function estimation using electro-encephalography for the patients with brain tumor managed by radiotherapy. Jpn. J. Clin. Radiol. (Rinsho Hoshasen), 44, 1163-1170. (Abstract in English)

Mariya, Y., Sekizawa, G., Matsuoka, Y., Seki, H. \& Sugawara, T. (2010) Outcome of stereotactic radiosurgery for patients with non-small cell lung cancer metastatic to the brain. J. Radiat. Res., 51, 333-342.

Mehta, M.P., Tsao, M.N., Whelan, T.J., Morris, D.E., Hayman, J.A., Flickinger, J.C., Mills, M., Rogers, C.L. \& Souhami, L. (2005) The American society for therapeutic radiology and oncology (ASTRO) evidence-based review of the role of radiosurgery for brain metastases. Int. J. Radiat. Oncol. Biol. Phys., 63, 37-46.

Radiation Therapy Oncology Group. Acute radiation morbidity scoring criteria. [Accessed January 6, 2011]
Radiation Therapy Oncology Group. RTOG/EORTC late radiation morbidity scoring schema. [Accessed January 6, 2011]

Schoutten, L.J., Rutten, J., Huveneers, H.A.M. \& Twijnstra, A. (2002) Incidence of brain metastases in a cohort of patients with carcinoma of the breast, colon, kidney, and lung and melanoma. Cancer, 94, 2698-2705.

Serizawa, T. (2009) Radiosurgery for metastatic brain tumors. Int. J. Clin. Oncol., 14, 289-298.

Shaw, E., Scott, C., Souhami, L., Dinapoli, R., Kline, R., Loeffler, J. \& Farnan, N. (2000) Single dose radiosurgical treatment of recurrent previously irradiated primary brain tumors and brain metastases: final report of RTOG protocol. Int. J. Radiat. Oncol. Biol. Phys., 47, 291-298.

Shuto, T., Fujino, H., Inomori, S. \& Nagano, H. (2004) Repeated gamma knife radiosurgery for multiple metastatic brain tumors. Acta Neurochir., 146, 989-993.

Sneed, P.K., Suh, J.H., Goetsch, S.J., Sanghavi, S.N., Chappel, R., Buatti, J.M., Regine, W.F., Weltman, E., King, V.J., Breneman, J.C., Sperduto, P.W. \& Mehta, M.P. (2002) A multiinstitutional review of radiosurgery alone vs. radiosurgery with whole brain radiotherapy as the initial management of brain metastases. Int. J. Radiat. Oncol. Biol. Phys., 53, 519526.

Sorensen, J.B., Hansen, H.H., Hansen, M. \& Dombernowsky, M. (1988) Brain metastases in adenocarcinoma of the lung: frequency, risk groups and prognosis. J. Clin. Oncol., 6, 14741480.

Wen, P.Y. \& Loeffler, J.S. (1999) Management of brain metastasis. Oncology, 13, 941-954.

Withers, H.R., Taylor, J.M.G. \& Maciejewski, B. (1988) Treatment volume and tissue tolerance. Int. J. Radiat. Oncol. Biol. Phys., 14, 751-759.

Yamanaka, K., Iwai, Y., Yasui, T., Nakajima, H., Komiyama, M., Nishikawa, M., Morikawa, T. \& Kishi, H. (1999) Gamma Knife radiosurgery for metastatic brain tumor: the usefulness of repeated Gamma Knife radiosurgery for recurrent cases. Stereotact. Funct. Neurosurg., 72 (Suppl. 1), 73-80. 